\title{
Conformational fitting of a flexible oligomeric substrate does not explain the enzymatic PET degradation
}

\author{
Ren Wei (ib) ${ }^{1,2 \star}$, Chen Song (10 ${ }^{3}$, Daniel Gräsing ${ }^{3}$, Tobias Schneider ${ }^{1}$, Pavlo Bielytskyi (1) ${ }^{3}$, Dominique Böttcher ${ }^{2}$, \\ Jörg Matysik (D) ${ }^{3}$, Uwe T. Bornscheuer (1) ${ }^{2} \&$ Wolfgang Zimmermann (1) ${ }^{1}$
}

ARISING FROM Joo et al. Nature Communications https://doi.org/10.1038/s41467-018-02881-1

oo et al. ${ }^{1}$ have recently reported a crystal structure of a polyethylene terephthalate (PET) hydrolyzing enzyme (IsPETase) from Ideonella sakaiensis which has been described able to metabolize PET at $30^{\circ} \mathrm{C}^{2}$. They proposed a PET degradation mechanism solely based on covalent computational docking of an oligomeric substrate-2-hydroxyethyl-(monohydroxyethyl terephthalate $)_{4}-\left(2-\mathrm{HE}(\mathrm{MHET})_{4}\right)$ into the substrate binding cleft of IsPETase without considering the motions and conformations of the PET polymer chain. Here we present a solid-state nuclear magnetic resonance (NMR) analysis of amorphous PET at the degradation temperature used by Joo et al. ${ }^{1}$, indicating that the highly stiff polymer chain can hardly resemble the suggested docking conformation of $2-\mathrm{HE}(\mathrm{MHET})_{4}$. In correlation with the PET degradation performance obtained at the same temperature, IsPETase is unlikely to follow the catalytic mechanism proposed by Joo et al. ${ }^{1}$, which requires simultaneous binding and interaction of all four MHET substrate moieties with the binding site of the enzyme.

While IsPETase can hydrolyze amorphous PET, it showed almost no activity against the crystalline PET polymer ${ }^{2}$. The OC-CO torsion angle $\Psi$ in the ethylene glycol (EG) units of amorphous and crystalline PET reveals distinct probability distributions ${ }^{3}$. According to the 2-HE(MHET $)_{4}$ docking conformation described by Joo et al. ${ }^{1}$, a trans $\left(t, \Psi_{t} \approx 180^{\circ}\right)$ content higher than $25 \%$ was obtained, considerably higher than the literature value of $14 \pm 5 \%$ obtained for amorphous PET at ambient temperature $^{3}$. In our study here, we determined the probability distribution of the OC-CO torsion angle $\Psi$ in a commercially available amorphous PET material and obtained a trans to gauche $\left(\Psi_{g} \approx \pm 70^{\circ}\right)$ ratio of $9: 91$ at $30^{\circ} \mathrm{C}$ (Fig. 1a), the temperature at which Joo et al. ${ }^{1}$ performed their PET degradation experiments ${ }^{1}$. This value is in good agreement with the literature ${ }^{3}$ but significantly lower than the $t / g$ ratio obtained in the 2-HE(MHET) $)_{4}$ docking conformation, suggesting that the latter conformation is rarely present in amorphous PET polymer chains. As a consequence, even if the target ester bond in $2-\mathrm{HE}(\mathrm{MHET})_{4}$ between subsite I and subsite IIa can be correctly accessed by the catalytic triad of the IsPETase (Fig. $2 \mathrm{~b}$ in Joo et al. ${ }^{1}$ ), residues in distal subsites IIb and IIc are unlikely to interact with the other two MHET moieties due to a more biased presence of gauche contents in the PET polymer. In a later publication dealing with the IsPETase structure ${ }^{4}$, Austin et al. ${ }^{4}$ have also shown induced fit docking conformations for a PET tetramer, which is equivalent to $2-\mathrm{HE}(\mathrm{MHET})_{4}$ used by Joo et al. ${ }^{1}$. For the wildtype IsPETase and a double mutant $(\mathrm{S} 238 \mathrm{~F} / \mathrm{W} 159 \mathrm{H})$ with increased PET hydrolyzing activity, different energetically most-favored conformations of the PET tetramer were demonstrated by Austin et al. ${ }^{4}$ : a neartrans conformer for the EG unit directly flanking the target ester bond in the wildtype enzyme and a gauche conformer in the double mutant. Nevertheless, in contrast to Joo et al. ${ }^{1}$, Austin et al. ${ }^{4}$ did not report on the questionable interactions of distal PET repeating units with surrounding residues in their hypothesis of substrate binding and catalysis.

Moreover, we examined the localized and large-scale cooperative main-chain motions in amorphous PET by magic-anglespinning (MAS) NMR methods. The ${ }^{1} \mathrm{H}-{ }^{13} \mathrm{C}$ dipolar coupling order parameters $S$ measured using 2D dipolar chemical shift correlation (DIPSHIFT) experiments were used to quantify the site-specific motions on a sub-microsecond timescale to describe the relative rigidity in a value range of 0 to $1^{5}$. Experimental data obtained with crystalline bis(2-hydroxyethyl) terephthalate (BHET) were used to define the rigid limit corresponding to $S=$ 1. At $30^{\circ} \mathrm{C}$, the dominant gauche conformer in $\mathrm{EG}$ units of amorphous PET showed an order parameter of $S_{\mathrm{CH}}=0.96$, indicating that the transition from gauche to trans conformation was highly restricted (Fig. 1b). Therefore, conformational changes in PET segments in order to perfectly fit the substrate binding cleft of IsPETase as described by Joo et al. ${ }^{1}$ cannot occur at $30^{\circ} \mathrm{C}$.

\footnotetext{
${ }^{1}$ Department of Microbiology and Bioprocess Technology, Institute of Biochemistry, Leipzig University, Johannisallee 21-23, D-04103 Leipzig, Germany.

2 Department of Biotechnology and Enzyme Catalysis, Institute of Biochemistry, University of Greifswald, Felix-Hausdorff-Str. 4, D-17487 Greifswald,

Germany. ${ }^{3}$ Institute of Analytical Chemistry, Leipzig University, Linnéstrasse 3, D-04103 Leipzig, Germany. *email: ren.wei@uni-greifswald.de
} 

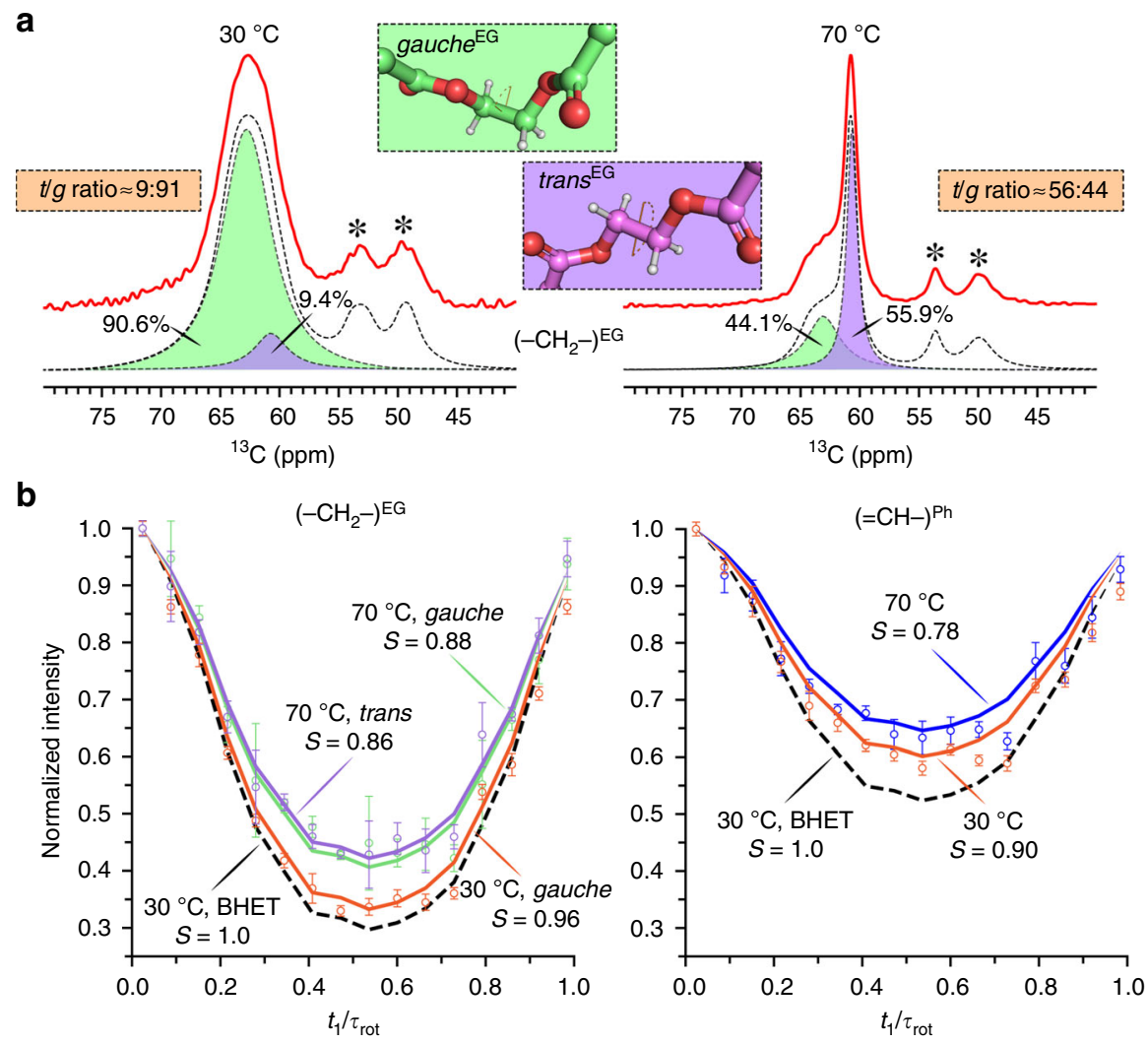

Fig. 1 MAS NMR of amorphous PET powder at $30^{\circ} \mathrm{C}$ and $70^{\circ} \mathrm{C}$. a ${ }^{13} \mathrm{C}$ spectra in the range of $40-80$ ppm showing the characteristic spectra for the EG carbons. The trans and gauche contents were quantified by fitting a Voigt function to the experimental spectra (red). The sum of the Voigt fits (shaded in purple and green, respectively, for trans and gauche conformers) is shown as dotted curve. The inset shows the EG fragment in the trans and gauche conformations. MAS sidebands are denoted by asterisks. $\mathbf{b}^{1} \mathrm{H}-{ }^{13} \mathrm{C}$ DIPSHIFT curves for phenylene and EG carbons acquired at a MAS rate of $8 \mathrm{kHz}$. The solid lines are the best-fit simulations and dashed lines represent the rigid limit. Experimental error bars were determined from the noise level. Order parameters $S$ from the measured dipolar couplings are given.

Nevertheless, we determined a more than $1 \%$ weight loss of the same amorphous PET film as a result of IsPETase-catalyzed hydrolysis following $24 \mathrm{~h}$ of incubation at $30^{\circ} \mathrm{C}$ (Fig. 2). Based on the molecular dynamics simulations shown in another study, amino acid residues and backbones involved in the substrate binding cleft of IsPETase are not allowed to move freely to fit any arbitrary rigid conformation of a PET polymer segment. Thus, Is PETase is likely to follow other binding and degradation mechanisms with regard to the PET hydrolysis at $30^{\circ} \mathrm{C}$ than those proposed by Joo et al. ${ }^{1}$.

PET has a glass transition temperature above $70^{\circ} \mathrm{C}^{7}$ which has been suggested as a more favorable reaction condition for enzymatic PET hydrolysis ${ }^{8,9}$. Yoshida et al. ${ }^{2}$ verified this by demonstrating a more than 100-fold higher release of UV-absorbing degradation products using LC-cutinase, a thermophilic homologous counterpart of IsPETase, against an amorphous PET sample than using IsPETase after an incubation time of $1 \mathrm{~h}$ at their individual optimal reaction conditions ${ }^{2}$. Similarly, we compared the degradation performance of both enzymes against the PET material used in this study and showed a more than 40 fold higher weight loss obtained by LC-cutinase after an incubation time of $24 \mathrm{~h}$ (Fig. 2). These differences in their PET degradation performance were considered to be strongly dependent on the polymer chain mobility and accessibility at different temperatures, as suggested previously also in another publication $^{8}$. As shown in Fig. 1, we observed slightly larger amplitudes of motions in EG units at $70^{\circ} \mathrm{C}$ indicated by the lower order parameters $S_{\mathrm{CH}}$ of 0.86 (trans) and 0.88 (gauche). As a consequence, the transition between trans and gauche conformers was allowed resulting in a $t / g$ ratio of $56: 44$, a significantly higher value than that obtained at $30^{\circ} \mathrm{C}$. This indicated a transition tendency of PET polymers from a less-ordered state to a moreordered one as a result of physical aging during the incubation at $70^{\circ} \mathrm{C}$, which is consistent with our recent publication ${ }^{10}$. Interestingly, the aromatic phenylene rings in amorphous PET exhibited lower order parameters with $S_{\mathrm{CH}}=0.90$ and 0.78 at $30^{\circ} \mathrm{C}$ and $70^{\circ} \mathrm{C}$, respectively, indicating that the phenylene units in PET are more prone to motions compared with the flanking rigid EG units at both temperatures. This is in agreement with a previous publication reporting a phenylene ring flip motion in amorphous PET on a millisecond-to-second timescale ${ }^{11}$. Therefore, we hypothesize that the weak interactions between the aromatic phenylene units and the surrounding hydrophobic amino acid residues are more likely to facilitate the substrate binding requested for the subsequent enzymatic hydrolysis of PET rather than the perfect accommodation of a certain conformation of a polymer segment, especially at an ambient temperature around $30^{\circ} \mathrm{C}$.

\section{Methods}

Solid-state NMR analysis of amorphous PET powder. Amorphous PET films (product no. ES301445) were purchased from Goodfellow Cambridge Ltd. (Huntingdon, UK) and ground in a cryomill in the presence of liquid nitrogen. Particles with diameters of less than $0.25 \mathrm{~mm}$ were obtained by sieving and then used in the solid-state NMR analysis. All CP/MAS (cross-polarization magic-angle spinning) NMR experiments were performed at $9.4 \mathrm{~T}$ with a Bruker AVANCE III NMR spectrometer equipped with a 4-mm double-resonance MAS probe (Rheinstetten, 


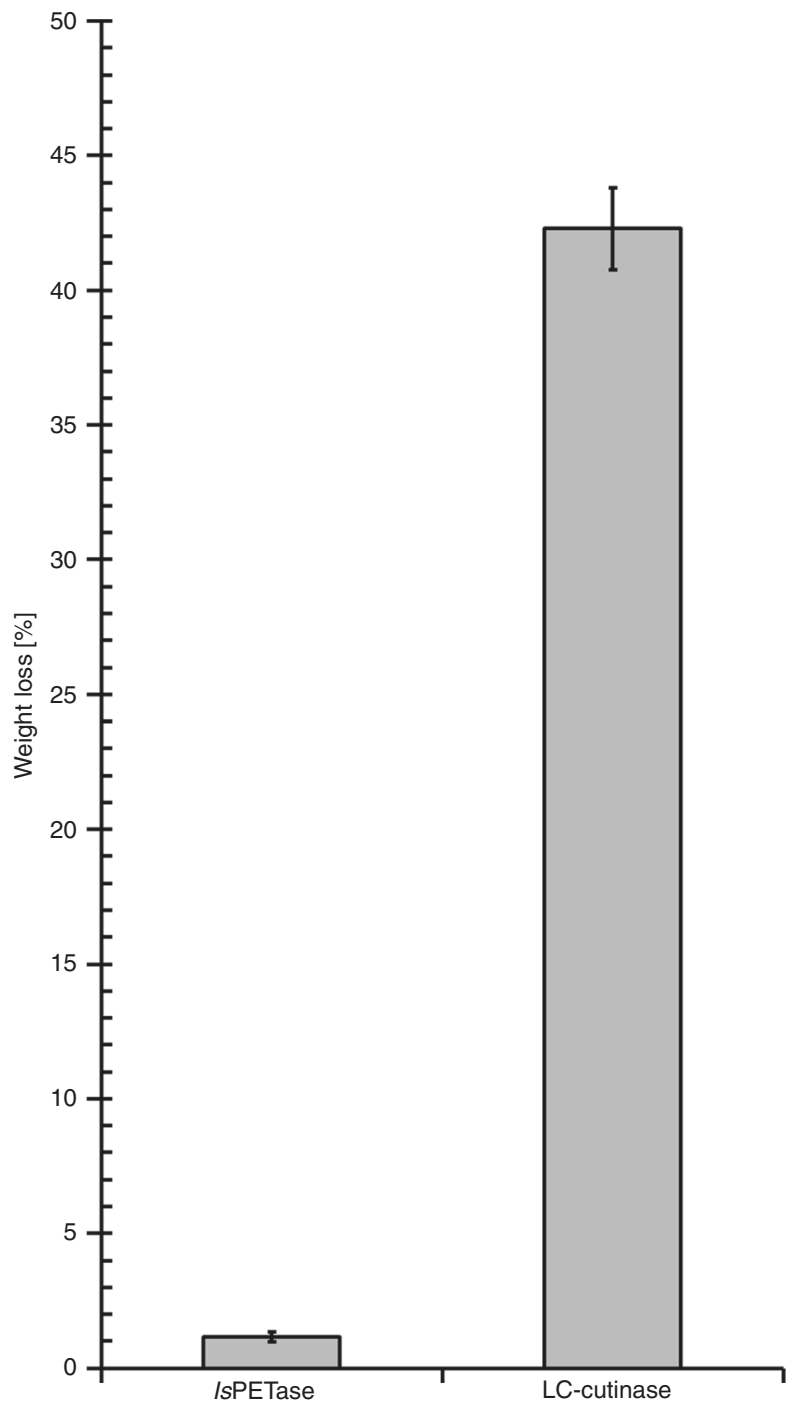

Fig. 2 Weight loss of an amorphous PET film $(\sim 45 \mathrm{mg})$ as a result of enzymatic hydrolysis. Incubation with IsPETase and LC-cutinase was carried out under agitation for $24 \mathrm{~h}$ at $30^{\circ} \mathrm{C}$ and $70^{\circ} \mathrm{C}$, respectively, i.e., at their optimal reaction conditions for $\mathrm{PET}$ degradation. Error bars indicate the standard deviation of triplet measurements.

Germany). A MAS rate of $8 \mathrm{kHz}$ was maintained throughout the experiments. The ${ }^{207} \mathrm{~Pb}$ NMR resonance of lead nitrate was used as the thermometer to calibrate the temperature over the sample volume of the in-situ MAS probe at $8 \mathrm{kHz}^{12}$. The MAS data were acquired respectively at 30 and $70^{\circ} \mathrm{C}$ by using two individual samples. Each fine-powdered PET sample ( $30 \mathrm{mg})$ was loaded into a $4 \mathrm{~mm} \mathrm{ZrO}_{2}$ MAS rotor with Vespel cap and incubated at their individual target temperatures in the magnet for $24 \mathrm{~h}$ prior to the data acquisition. Optimized ${ }^{1} \mathrm{H}$ and ${ }^{13} \mathrm{C} 90^{\circ}$ pulse lengths were 2.5 and $3.0 \mu \mathrm{s}$, respectively. The CP/MAS spectra were recorded with 2512 scans and a relaxation delay of $4 \mathrm{~s}$, with optimized spin-lock pulses to satisfy both Hartmann-Hahn (HH) $n= \pm 1$ matching conditions, with $66 \mathrm{kHz}$ effective ${ }^{13} \mathrm{C}$ radio frequency (r.f.) lock field and $100-70 \%$ ramp on the ${ }^{1} \mathrm{H}$ channel. Decomposition of methylene carbon resonances from the EG units provided direct quantification for the $t / g$ ratio. The contact time was $2 \mathrm{~ms} .{ }^{1} \mathrm{H}-{ }^{13} \mathrm{C}$ order parameters were measured using the 2D DIPSHIFT experiments 5 . The experiments were conducted with 256 scans and a recycle delay of $4 \mathrm{~s} .{ }^{1} \mathrm{H}-1{ }^{1} \mathrm{H}$ homonuclear dipolar decoupling was accomplished with phase-modulated Lee-Goldburg (PMLG) approach ${ }^{13}$. The PMLG block consists of 10 pulses with the following phases: $339.22^{\circ}, 297.65^{\circ}, 256.08^{\circ}, 214.51^{\circ}, 172.94^{\circ}, 352.94^{\circ}, 34.51^{\circ}, 76.08^{\circ}, 117.65^{\circ}$, and $159.22^{\circ}$ ( $\mathrm{m} 5 \mathrm{~m}$ shape in TopSpin library, Bruker). The PMLG5-optimized pulse was $2.07 \mu$ s and the r.f. decoupling field was set to $80 \mathrm{kHz}$. The PMLG scaling factor of 0.5 was determined based on adamantane $J$-splitting. For all experiments, sweptfrequency two-pulse phase-modulation $\left(\mathrm{SW}_{\mathrm{f}}\right.$ - TPPM) heteronuclear decoupling ${ }^{14}$ with a r.f. field of $100 \mathrm{kHz}$ was used during the acquisition. ${ }^{13} \mathrm{C}$ chemical shifts were externally referenced to the $\mathrm{C}(=\mathrm{O}) \mathrm{O}^{-}$signal of solid tyrosine $\cdot \mathrm{HCl}$ at $172.1 \mathrm{ppm}$. The DIPSHIFT dephasing curves were simulated using the SIMPSON program ${ }^{15}$ The rigid-limit values for both $\mathrm{CH}$ and $\mathrm{CH}_{2}$ spin systems were obtained by fitting the experimental curves of crystalline BHET in a site-specific manner. Spectral fitting was conducted with the MestReNova 12.0.0 program (Mestrelab Research, Santiago de Compostela, Spain).

Enzymatic hydrolysis of amorphous PET films. A codon-optimized synthetic gene encoding IsPETase lacking the $\mathrm{N}$-terminal signal sequence containing 27 residues was ordered from Genscript (Piscataway, USA) and subcloned into the pET-21b vector (Novagen, San Diego, USA). The recombinant IsPETase containing a C-terminal $\mathrm{His}_{6}$-tag was expressed in Escherichia coli Shuffle T7 Express (New England Biolabs GmbH, Frankfurt am Main, Germany). Briefly, $E$. coli cells were grown at $30^{\circ} \mathrm{C}$ to an optical density $\left(\mathrm{OD}_{600}\right)$ of 1 , followed by induction in the presence of $0.1 \mathrm{mM} \mathrm{IPTG}$ at $16^{\circ} \mathrm{C}$ for more than $12 \mathrm{~h}$, and then purified by immobilized metal ion chromatography (IMAC) using TALON metal affinity resin (Takara Bio Europe, Saint-Germain-en-Laye, France) to homogeneity. LC-cutinase was recombinantly expressed in E. coli BL21(DE3) and purified by IMAC to homogeneity using Ni-NTA (Qiagen, Hilden, Germany) as previously described ${ }^{16} .5 \mu \mathrm{g}$ of IsPETase and $50 \mu \mathrm{g}$ of LC-cutinase were used to degrade a piece of amorphous PET film (Goodfellow Cambridge Ltd., $3 \times 0.5 \mathrm{~cm}^{2}, \sim 45 \mathrm{mg}$ ) using the optimal degradation conditions for the respective enzymes ${ }^{2,16}$. Briefly, Is PETase required $50 \mathrm{mM} \mathrm{Na}_{2} \mathrm{HPO}_{4}-\mathrm{HCl}$ at $\mathrm{pH}=7$ while LC-cutinase required $1 \mathrm{M} \mathrm{K}_{2} \mathrm{HPO}_{4} / \mathrm{KH}_{2} \mathrm{PO}_{4}$ at $\mathrm{pH}=8$. Degradation was performed by shaking the reaction vials on a thermoshaker TS1 (Biometra, Göttingen, Germany) at $1000 \mathrm{rpm}$ for $24 \mathrm{~h}$ at $30^{\circ} \mathrm{C}$ for IsPETase and at $70^{\circ} \mathrm{C}$ for LCcutinase. The reaction was stopped by cooling the samples on ice. PET films were washed sequentially with $0.1 \%$ aqueous SDS, ethanol and ultrapure water and then dried at $50^{\circ} \mathrm{C}$ for $48 \mathrm{~h}$ before subjected to gravimetric weight loss determination. Degradation experiments using higher IsPETase concentrations up to $50 \mu \mathrm{g}$ enzyme and lower LC-cutinase amount down to $5 \mu \mathrm{g}$ enzyme were also prepared as control samples which led to significantly lower weight losses of the PET films (data not shown) than using the enzyme amounts mentioned above in main text.

\section{Data availability}

The datasets generated during and/or analyzed during the current study are available from the corresponding author on reasonable request.

Received: 14 August 2018; Accepted: 4 November 2019; Published online: 06 December 2019

\section{References}

1. Joo, S. et al. Structural insight into molecular mechanism of poly(ethylene terephthalate) degradation. Nat. Commun. 9, 382 (2018).

2. Yoshida, S. et al. A bacterium that degrades and assimilates poly(ethylene terephthalate). Science 351, 1196-1199 (2016).

3. Schmidt-Rohr, K., Hu, W. \& Zumbulyadis, N. Elucidation of the chain conformation in a glassy polyester, PET, by two-dimensional NMR. Science 280, 714-717 (1998).

4. Austin, H. P. et al. Characterization and engineering of a plastic-degrading aromatic polyesterase. Proc. Natl Acad. Sci. USA 115, E4350-E4357 (2018).

5. deAzevedo, E. R. et al. Intermediate motions as studied by solid-state separated local field NMR experiments. J. Chem. Phys. 128, 104505 (2008).

6. Fecker, T. et al. Active site flexibility as a hallmark for efficient PET degradation by I. sakaiensis PETase. Biophy. J. 114, 1302-1312 (2018).

7. Alves, N. M., Mano, J. F., Balaguer, E., Meseguer Duenas, J. M. \& Gomez Ribelles, J. L. Glass transition and structural relaxation in semi-crystalline poly (ethylene terephthalate): a DSC study. Polymer 43, 4111-4122 (2002).

8. Ronkvist, Ãs. M., Xie, W., Lu, W. \& Gross, R. A. Cutinase-catalyzed hydrolysis of poly(ethylene terephthalate). Macromolecules 42, 5128-5138 (2009).

9. Wei, R. \& Zimmermann, W. Biocatalysis as a green route for recycling the recalcitrant plastic polyethylene terephthalate. Microb. Biotechnol. 10, 1302-1307 (2017).

10. Wei, R. et al. Biocatalytic degradation efficiency of postconsumer polyethylene terephthalate packaging determined by their polymer microstructures. $A d v$. Sci. 6, 1900491 (2019)

11. Choudhury, R. P., Lee, J. S., Kriegel, R. M., Koros, W. J. \& Beckham, H. W. Chain dynamics in antiplasticized and annealed poly(ethylene terephthalate) determined by solid-state NMR and correlated with enhanced barrier properties. Macromolecules 45, 879-887 (2012).

12. Guan, X. \& Stark, R. E. A general protocol for temperature calibration of MAS NMR probes at arbitrary spinning speeds. Solid State Nucl. Mag. 8, 74-76 (2010). 
13. Vinogradov, E., Madhu, P. K. \& Vega, S. Phase modulated Lee-Goldburg magic angle spinning proton nuclear magnetic resonance experiments in the solid state: a bimodal Floquet theoretical treatment. J. Chem. Phys. 115, 8983-9000 (2001).

14. Thakur, R. S., Kurur, N. D. \& Madhu, P. K. Swept-frequency two-pulse phase modulation for heteronuclear dipolar decoupling in solid-state NMR. Chem. Phys. Lett. 426, 459-463 (2006).

15. Bak, M., Rasmussen, J. T. \& Nielsen, N. C. SIMPSON: a general simulation program for solid-state NMR spectroscopy. J. Magn. Reson. 147, 296-330 (2000).

16. Schmidt, J. et al. Effect of Tris, MOPS, and phosphate buffers on the hydrolysis of polyethylene terephthalate films by polyester hydrolases. FEBS Open Bio 6, 919-927 (2016).

\section{Author contributions}

R.W. and C.S. designed the experiments; C.S., D.G., and P.B. performed the solid-state NMR analysis and the corresponding evaluation; T.S. and D.B. carried out the cloning, expression and purification of recombinant polyester hydrolases; T.S. and R.W. performed and evaluated the enzymatic PET hydrolysis; R.W., J.M., U.B., and W.Z. supervised the study; R.W. and C.S. wrote the manuscript; U.B., W.Z., D.B., and J.M. edited the manuscript.

\section{Competing interests}

The authors declare no competing interests.

\section{Additional information}

Correspondence and requests for materials should be addressed to R.W.

Peer review information Nature Communications thanks the anonymous reviewers for their contribution to the peer review of this work.

Reprints and permission information is available at http://www.nature.com/reprints

Publisher's note Springer Nature remains neutral with regard to jurisdictional claims in published maps and institutional affiliations.

\begin{abstract}
(c) (1)
Open Access This article is licensed under a Creative Commons Attribution 4.0 International License, which permits use, sharing, adaptation, distribution and reproduction in any medium or format, as long as you give appropriate credit to the original author(s) and the source, provide a link to the Creative Commons license, and indicate if changes were made. The images or other third party material in this article are included in the article's Creative Commons license, unless indicated otherwise in a credit line to the material. If material is not included in the article's Creative Commons license and your intended use is not permitted by statutory regulation or exceeds the permitted use, you will need to obtain permission directly from the copyright holder. To view a copy of this license, visit http://creativecommons.org/ licenses/by/4.0/.
\end{abstract}

(C) The Author(s) 2019 